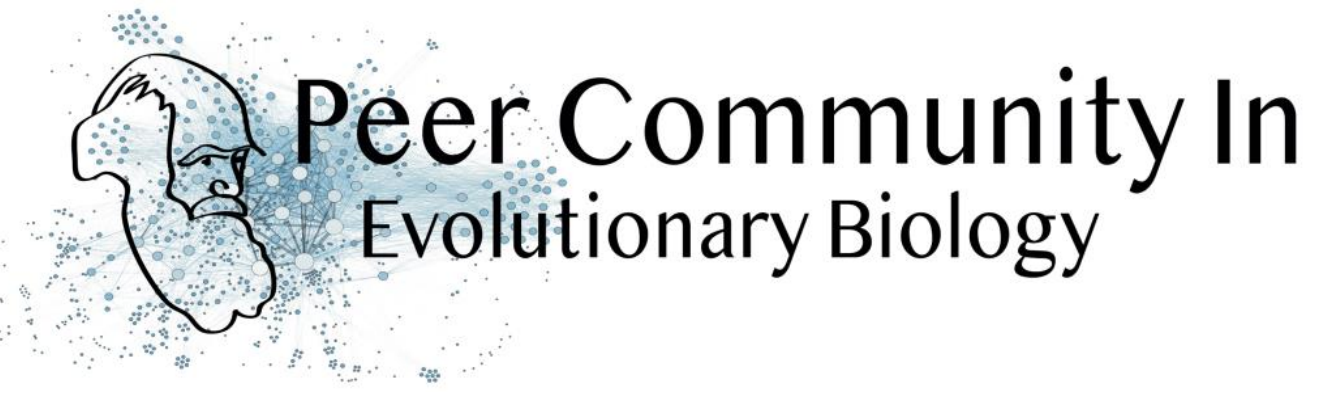

\title{
Habitat variation of wild clownfish population shapes selfrecruitment more than genetic effects
}

Philip Munday based on reviews by Juan Diego Gaitan-Espitia and Loeske Kruuk

\section{Open Access}

Published: 7 October 2019

Copyright: This work is licensed under the Creative Commons Attribution-NoDerivatives 4.0 International License. To view a copy of this license, visit

http://creativecommons.org/licen ses/by-nd/4.0/

\section{A recommendation of:}

Océane C. Salles, Glenn R. Almany, Michael L. Berumen, Geoffrey P. Jones, Pablo Saenz-Agudelo, Maya Srinivasan, Simon Thorrold, Benoit Pujol, Serge Planes. Strong habitat and weak genetic effects shape the lifetime reproductive success in a wild clownfish population (2019), Zenodo, 3476529, ver. 3 peerreviewed and recommended by Peer Community in Evolutionary Biology. 10.5281/zenodo.3476529

Submitted: 01 October 2018, Recommended: 30 September 2019 Cite this recommendation as:

Philip Munday (2019) Habitat variation of wild clownfish population shapes selfrecruitment more than genetic effects. Peer Community in Evolutionary Biology, 100082.

10.24072/pci.evolbiol.100082

Estimating the genetic and environmental components of variation in reproductive success is crucial to understanding the adaptive potential of populations to environmental change. To date, the heritability of lifetime reproductive success (fitness) has been estimated in a handful of wild animal population, mostly in 


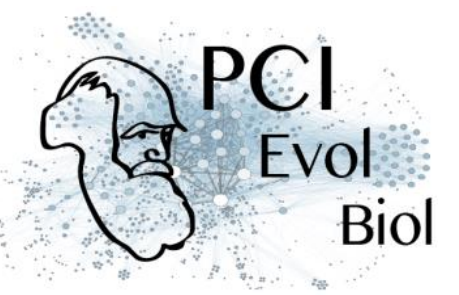

mammals and birds, but has never been estimated for a marine species. The primary reason that such estimates are lacking in marine species is that most marine organisms have a dispersive larval phase, making it extraordinarily difficult to track the fate of offspring from one generation to the next. In this study, Salles et al. [1] use an unprecedented 10 year data set for a wild population of orange clownfish (Amphiprion percula) to estimate the environmental, maternal and additive genetic components of life time reproductive success for the selfrecruiting portion of the local population. Previous studies show that over $50 \%$ of juvenile clownfish recruiting to the population of clownfish at Kimbe Island (Kimbe Bay, PNG) are natal to the population. In other words, $>50 \%$ of the juveniles recruiting to the population at Kimbe Island are offspring of parents from Kimbe Island. The identity and location of every adult clownfish in the Kimbe Island population was tracked over 10 years. At the same time newly recruiting juveniles were collected at regular intervals (biennially) and their parentage assigned with high confidence by 22 polymorphic microsatellite loci. Salles et al. then used a pedigree comprising 1735 individuals from up to 5 generations of clownfish at Kimbe Island to assess the contribution of every breeding pair of clownfish to self-recruitment within the local population. Because clownfish are site attached and live in close association with a host sea anemone, it was also possible to examine the contribution of reef location and host anemones species (either Heteractis magnifica or Stichodactyla gigantea) to reproductive success within the local population. The study found that breeders from the eastern side of Kimbe Island, and mostly inhabiting S. gigantea sea anemones, produced more juveniles that recruited to the local population than breeders from other location around the island, or inhabiting $H$. magnifica. In fact, host anemone species and geographic location explained about $97 \%$ of the variance in reproductive success within the local population (i.e. excluding successful recruitment to other populations). By contrast, maternal and additive genetic effects explained only $1.9 \%$ and $1.3 \%$ of the variance, respectively. In other words, reef location and the species of host anemone inhabited had an overwhelming influence on the longterm contribution of breeding pairs of clownfish to replenishment of the local population. This overwhelming effect of the local habitat on reproductive success means that the population is potentially susceptible to rapid environmental 


\section{Biol}

changes - for example if $S$. giganta sea anemones are disproportionately susceptible to global warming, or reef habitats on the eastern side of the island are more susceptible to disturbance. By contrast, the small component of additive genetic variance in local reproductive success translated into low heritability and evolvability of lifetime reproductive success within the local population, as predicted by theory [2] and observed in some terrestrial species. Consequently, fitness would evolve slowly to environmental change. Establishing the components of variation in fitness in a wild population of marine fishes is an astonishing achievement, made possible by the unprecedented long-term individual-level monitoring of the entire population of clownfish at Kimbe Island. A next step in this research would be to include other clownfish populations that are demographically and genetically connected to the Kimbe Island population through larval dispersal. It would be intriguing to establish the environmental, maternal and additive genetic components of reproductive success in the dispersing part of the Kimbe Island population, to see if this potentially differs among breeders who contribute more or less to replenishment within the local population.

\section{References}

[1] Salles, O. C., Almany, G. R., Berumen, M.L., Jones, G. P., Saenz-Agudelo, P., Srinivasan, M., Thorrold, S. R., Pujol, B., Planes, S. (2019). Strong habitat and weak genetic effects shape the lifetime reproductive success in a wild clownfish population. Zenodo, 3476529, ver. 3 peer-reviewed and recommended by Peer Community In Evolutionary Biology. doi: 10.5281/zenodo.3476529

[2] Fisher, R.A. (1930). The genetical theory of natural selection. Clarendon Press, Oxford, U.K.

\section{Revision round \#2}

2019-07-01 
Dear Drs Salles and Pujol,

Thank you for resubmitting your manuscript, with the revised title "Determinants of lifetime reproductive success in a wild clownfish population: strong habitat and weak genetic effects". Thanks also for your careful and thorough attention to the comments and questions raised by the two referees on the original ms. In general I am satisfied that you have satisfactorily addressed their concerns and suggestions and the $\mathrm{ms}$ is significantly improved as a result. However, the major change you have made in framing the ms around lifetime reproductive success (LRS) instead of self-recruitment throws up a major concern for me. In the original ms, entitled "Habitat variation of wild clownfish population shapes selfrecruitment more than genetic effects" it was very clear that you were investigating the genetic and environmental components of self-recruitment in the study population of fishes at Kimbe Island. Rephrasing this to mean LRS opens a whole new can of worms that needs to be considered.

My overriding concern about stating that you are testing the heritability and evolvability of LRS is that you are only testing this is the fish that return to their natal population. My understanding of the study system is that roughly about $50 \%$ of juveniles recruit to the natal population and about $50 \%$ of the juveniles recruit to other reefs, up to about $50 \mathrm{~km}$ away. If that's the case you are only dealing with about half the LRS on average. There are several important issues to follow from this observation:

1) You will underestimated LRS because (presumably) most breeders will have a significant component of successful recruitment (reproductive success) beyond the sampled population at Kimbe Island. More importantly, there is also a risk that your estimates of LRS could be biased if some breeders have much higher reproductive success through dispersers compared with self-recruiters. Consider a situation where the breeders in poor habitats for self-recruitment at Kimbe Island are also the same breeders that have very high success with recruitment of dispersing juveniles at other reefs. This would bias your estimates of LRS and could inflate your estimate of the environmental component of variation in LRS at the expense of genetic components of variance in LRS. 
2) It is also possible that the genetic versus environmental components of successful recruitment success are different for the portion of the population that disperse to other locations compared with those that recruit to the natal population. Consider for example if the success of dispersing juveniles has a large additive genetic component. Excluding the $50 \%$ of the successful reproduction that disperse could seriously bias the estimation of heritability of LRS. In other words, your estimate of genetic and environmental components of variation in LRS must assume that the same proportions extend to the $50 \%$ of the population that is not included in the analysis.

Based on the responses to reviewer comments, your change from referring to heritability and evolvability of self-recruitment in the original ms to LRS in the revised ms seems to be in response to Loeske Kruuk's 3rd major comment. In that comments she noted that what marine ecologists call self-recruitment would be called local recruitment in other fields. It seems to me that this was just a matter for clarification and I don't think she was necessarily saying you should change the story to argue that you were measuring LRS, even if that is what many of the examples in Table 1 are reporting. An extension of this issue is that many of the examples given in Table 1 probably are indeed able to get a relatively unbiased estimate of LRS because the terrestrial study populations are island bound, and therefore, do not suffer from the inherent problem in marine fish population where there is widespread dispersal beyond the study population and much of the successful recruitment (reproductive success) happens outside the area that can be sampled.

I would like you to consider these concerns and either revise the ms accordingly, or provide a reasoned and detailed explanation as to why they are not relevant. If you revise the ms, my preference would be to revert to using the original dialogue around testing the genetic and environmental components of self (local) recruitment. If you retain the LRS dialogue, the assumptions and limitations will need to be very explicitly and clearly stated early in the ms (i.e. in the introduction). Either way, I think the title must change as it's misleading in its current formulation. 
I reiterate that I think this is an excellent and important study and I make these comments in the interests of seeing the most robust and convincing ms presented.

\section{Other comments}

Line 127. Delete "both"

Line 154. Delete "the" before "anemone"

Line 171. Please clarify what you mean by "before settling on an anemone that may or may not be in the population"? I think you mean settling to an anemone, either at their natal location (Kimbe Island) or elsewhere.

Line 199-200. It would be clearer if these two sentences were merged to read "We kept assignments to known parental pairs, but rejected assignments to single adults." \&nbsp

Additional requirements of the managing board: As indicated in the 'How does it work?' section and in the code of conduct, please make sure that: -Data are available to readers, either in the text or through an open data repository such as Zenodo (free), Dryad (to pay) or some other institutional repository. Data must be reusable, thus metadata or accompanying text must carefully describe the data.

-Details on quantitative analyses (e.g., data treatment and statistical scripts in R, bioinformatic pipeline scripts, etc.) and details concerning simulations (scripts, codes) are available to readers in the text, as appendices, or through an open data repository, such as Zenodo, Dryad or some other institutional repository. The scripts or codes must be carefully described so that they can be reused. -Details on experimental procedures are available to readers in the text or as appendices. -Authors have no financial conflict of interest relating to the article. The article must contain a "Conflict of interest disclosure" paragraph before the reference section containing this sentence: "The authors of this preprint declare that they have no financial conflict of interest with the content of this article." If appropriate, this disclosure may be completed by a sentence indicating that some of the authors are $\mathrm{PCI}$ recommenders: "XXX is one of the $\mathrm{PCI} X X X$ recommenders." 


\section{Revision round \#1}

2018-11-14

Dear Dr Salles,

I now have two expert reviews on your preprint. As you will see, both reviewers were very positive about the study and the amazing data set that underpins it. They also had some important and insightful comments that were offered in the spirit of further improving the manuscript. There are some important details about the study missing from the manuscript, clarifications are needed in some parts of the text, and also some statistical issues to consider. I have carefully read the reviews and concur with the reviewers' assessments. I therefore ask that you to revise the manuscript to account for these comments and suggestions and submit an updated preprint. Please also provide a detailed point-by-point response to the reviewers' comments. I will then be able to further consider the preprint for recommendation.

In regard to Reviewer 1's comments about terminology, I am not overly concerned about the term "self-recruitment" because it is widely used and understood in marine biology, but I do think that it needs to be carefully defined at first use so there is no confusion for other readers and synonymy with other terms is apparent.

I look forward to receiving your revised preprint.

Regards,

Philip Munday

Preprint DOI: https://doi.org/10.5281/zenodo.1435637

Reviewed by Loeske Kruuk, 2018-10-03 01:47 


\section{Reviewed by Juan Diego Gaitan-Espitia, 2018-10-03 02:40}

\section{Review PCI 2018}

The manuscript "Habitat variation of wild clownfish population shapes selfrecruitment more than genetic effects" by Océane C. Salles et al explores two interesting questions in evolutionary ecology: 1) What's the role/contribution of self-recruitment to local population replenishment?; and 2) to what extent additive genetic, maternal and environmental (i.e., habitat) variation contribute to phenotypic variation in components of self-recruitment?. The logic behind this study is that environmental changes (warming, habitat loss/fragmentation), are causing global declines in reef fish populations by affecting larval development and dispersal capacity. The main demographic/ecological consequence of this is (will be) a decrease in the ability of reef fish populations to replenish themselves and therefore the contribution of self-recruitment is expected to increase. By assessing the influence of genetic and environmental components of phenotypic variation on self-recruitment, the authors aimed to reveal the potential of fish populations to respond to selection on this trait. For this, Salles and co-authors used a unique (and amazing!) dataset (pedigree) of 5 generations of the orange clownfish within a 10-year time frame. As expected, the authors found extremely low to negligible additive genetic variation for self-recruitment, and most of the variation in this trait was explained by the habitat (quality, availability, distribution of host-anemones). Overall, the manuscript is very interesting, well designed/written and easy to follow, with a great potential for publication in a good journal. The Ms includes a good theoretical framework, hypothesis and methods are nicely described. Results are fully detailed and discussion is clear. However, before recommending this MS, I invite the authors to address the following comments and clarify some points that are confusing (at least for me). I hope these comments and suggestions will serve to improve the quality and impact of this MS:

Intro (Lines 54-57): In general, I got the idea of the importance of self-recruitment. However, it is still difficult to see the main effect of this trait on Darwinian fitness (at the individual level). The authors explained the existence of self-recruitment at 
different levels in this section, but did not explain whether this trait shows variation within/among populations, or its connection with fitness. The question in Line 57 is valid but needs to clarify these points before moving in that direction. There is an implicit assumption (reading between lines) across the MS that this trait is an adaptation shaped by past selection. I really can't see so far the adaptive role and fitness connection of this trait in the current version of the MS. Why should we expect evolutionary adaptive change in self-recruitment? Is it because directional selection is acting on self-recruitment per se? or because correlational selection? Making a clear connection of self-recruitment to fitness will help to visualise this issue (it is assumed along the MS but it is not clear).

Lines 57-58: What do you mean with "at the individual level"? individual species? Populations? Or organisms within populations?.

For the general audience it would be good to have a clear distinction between "the ability of populations to replenish themselves" and "self-recruitment", which in principle sound related. There is a good definition of self-recruitment in Lines 51-52, but it would be easier for the reader to see something similar for the first concept.

Line 72: add "additive" to "genetic variation". The presence of other sources of genetic variation doesn't play the say role in adaptive evolution.

Lines 76-78/86-87: Why do you expect very low levels of genetic variation and contribution to fitness (Va and maternal) for self-recruitment? I am not talking about a potential cause (78-81) but the reasoning of these expectations.

Lines 78-81: I understand the idea of this sentence but can't follow its connection with the trait of interest here. What is a "less fit genetic variant" in terms of selfrecruitment? Past selection and evolutionary equilibrium... are there other more plausible options?? (e.g., environmental influenced, no real effect on fitness)

Lines 88-89: Not only these factors as the presence/absence of genetic correlations and trade-offs are relevant for evolutionary responses.

Lines 90-91: Phenotypic plasticity can also enhance microevolutionary responses to selection (e.g., genetic assimilation). 
Methods: The study seems to be well designed and the statistical analysis seems valid, as well as the mixed-effect approach that the authors used.

Results: This section is a bit odd. There are parts that seem more relevant for the intro or discussion than for results (e.g., Lines 235-242). In addition, it looks repetitive when the authors report the values in the text but also in tables and figures of the same section. Minor comment here: try to keep uniform "additive/Additive" “maternal/Maternal” (e.g., Lines 230-233).

Discussion: Lines 291-292: Assuming that selection is in fact acting on this trait. Moreover, here you are reporting results.

Lines 316-326: migration was indeed a big component of population renewal (44\%). However, selection against migrants may also be involved in the low Va detected, especially when migrants are from very contrasting habitats (See Nosil et al., 2005; Hendry et al., 2002). Moreover, it is true that gene flow is expected to increase genetic variation. However, this is mainly neutral genetic variation which is not always linked to additive genetic variation, and therefore it is selectively neutral and tells us nothing about the adaptive or evolutionary potential of a population or a species (Holderegger et al., 2006).

Line 327, 329-: But you showed here that maternal effects do not contribute to the genetic variance of self-recruitment.

In some parts of the text, there are jumps in the focus to climate change instead of discussing to a greater extend the low Va of this trait, the existence of selection (type, direction, etc), and the potential for genetic correlations influencing the adaptive landscape. The single population focus is also a limitation. Trying to move your conclusions to a broader scale may help to understand the evolutionary dynamics of self-recruitment in your study system.

References:

Hendry, A.P., Taylor, E.B. \& McPhail, J.D. 2002. Adaptive divergence and the balance between selection and gene flow: lake and stream stickleback in the Misty system. Evolution 56: 1199-1216. 


\section{Biol}

Holderegger, R., Kamm, U., \& Gugerli, F. (2006). Adaptive vs. neutral genetic diversity: implications for landscape genetics. Landscape Ecology, 21(6), 797-807. Nosil, P., Vines, T. H., \& Funk, D. J. (2005). Reproductive isolation caused by natural selection against immigrants from divergent habitats. Evolution, 59(4), 705-719.

\section{Author's reply:}

Please see response to reviewers.

Access to documents: Please do not hesitate to request access to the files on the zenodo repository https://doi.org/10.5281/zenodo.3248745 where they are available in .doc format (I will send the documents by email to the recommender too).

No track changes here: The revised manuscript does not include text modifications in a different colour because substantial reorganisation and revision was done. However, the response to reviewers references the lines where changes were made according to comments

Looking forward to hearing from you Regards, Benoit

Download author's reply (PDF file) 